\title{
Test of discrete symmetries with neutral kaons at KLOE-2
}

Antonio Di Domenico*

on behalf of the KLOE-2 collaboration

Dipartimento di Fisica, Sapienza Università di Roma, and INFN Sezione di Roma, Rome, Italy

E-mail: antonio.didomenico@romal.infn.it

The KLOE-2 experiment at the INFN Laboratori Nazionali di Frascati (LNF) is currently taking data at the $e^{+} e^{-}$DAФNE collider which is implementing an innovative collision scheme based on a crab-waist configuration. An integrated luminosity of $\sim 4 \mathrm{fb}^{-1}$ has been already collected by KLOE-2, and $\geq 5 \mathrm{fb}^{-1}$ is in total expected by Spring 2018. KLOE-2 represents the continuation of KLOE with an upgraded detector and an extended physics program which includes neutral kaon interferometry and test of discrete symmetries among the main topics.

Entangled neutral kaon pairs produced at DA $\Phi N E$ are a unique tool to test discrete symmetries and quantum coherence at the utmost sensitivity, in particular strongly motivating the experimental searches of possible CPT violating effects, which would constitute an unambiguous signal of a New Physics framework.

The status of the latest ongoing analyses of KLOE/KLOE-2 data is reviewed. In particular a new preliminary measurement of the $\mathrm{K}_{\mathrm{S}}$ semileptonic charge asymmetry and related CPT tests are presented.

The European Physical Society Conference on High Energy Physics

5-12 July, 2017

Venice

${ }^{*}$ Speaker. 


\section{Introduction}

DA $\Phi$ NE, the Frascati $\phi$-factory, is an $e^{+} e^{-}$collider working at a center of mass energy of $\sqrt{s} \sim 1020 \mathrm{MeV}$ [1], corresponding to the peak of the $\phi$ resonance. The KLOE experiment at DAФNE completed its first data taking campaign in March 2006 with a total integrated luminosity of $\sim 2.5 \mathrm{fb}^{-1}$, corresponding to a production of $\sim 7.5 \times 10^{9} \phi$-mesons and $\sim 2.5 \times 10^{9} \mathrm{~K}^{0} \overline{\mathrm{K}}^{0}$ pairs.

The KLOE detector is a $4 \pi$ detector setup, which is able to measure both charged and neutral particles. It consists of a large volume drift chamber [2], which provides excellent momentum and vertex reconstruction for charged particles, and a barrel shaped electromagnetic calorimeter with two end-caps [3], made from lead and scintillating fibers, which surrounds the drift chamber. The energy deposits of charged and neutral particles in the calorimeter are measured with very good time resolution, allowing particle identification with time-of-flight (TOF) techniques. Drift chamber and calorimeter are enclosed in a superconducting solenoid, providing an axial $0.52 \mathrm{~T}$ magnetic field.

After the KLOE run, DAФNE has been upgraded implementing an innovative collision scheme based on a crab-waist configuration [4, 5]. The KLOE-2 experiment [6], aiming to continue and extend the physics program of its predecessor, started its data-taking in November 2014 at the upgraded DA $\Phi N E$ and with an improved KLOE detector. An integrated luminosity of $\sim 4 \mathrm{fb}^{-1}$ has been already collected, and a total of at least $5 \mathrm{fb}^{-1}$ is expected to be achieved by Spring 2018 . The KLOE-2 physics program has been described in detail in Ref. [6] and among the main issues includes neutral kaon interferometry and tests of discrete symmetries and quantum mechanics. The upgrade of the KLOE detector consisted in the addition of (i) an inner tracker based on cylindrical GEM technology for the improvement of tracking and decay vertex resolution close to the interaction point (IP), (ii) a $e^{ \pm}$tagging system for the $\gamma \gamma$ physics, and (iii) two calorimeters in the final focusing region to improve acceptance and efficiency for photons coming from the IP and neutral kaon decays inside the detector volume.

\section{Entangled neutral K mesons at KLOE-2}

At a $\phi$-factory neutral kaons are produced in the entangled state:

$$
|i\rangle=\frac{1}{\sqrt{2}}\left\{\left|\mathrm{~K}^{0}\right\rangle\left|\overline{\mathrm{K}}^{0}\right\rangle-\left|\overline{\mathrm{K}}^{0}\right\rangle\left|\mathrm{K}^{0}\right\rangle\right\}=\frac{\mathscr{N}}{\sqrt{2}}\left\{\left|\mathrm{~K}_{\mathrm{S}}\right\rangle\left|\mathrm{K}_{\mathrm{L}}\right\rangle-\left|\mathrm{K}_{\mathrm{L}}\right\rangle\left|\mathrm{K}_{\mathrm{S}}\right\rangle\right\},
$$

with $\mathscr{N} \simeq 1$ a normalization factor.

The observable quantity is the double differential decay rate of the state $|i\rangle$ into decay products $f_{1}$ and $f_{2}$ at proper times $t_{1}$ and $t_{2}$, respectively. After integration on $\left(t_{1}+t_{2}\right)$ at fixed time difference $\Delta t=t_{1}-t_{2}$, the decay intensity can be written as follows [7]:

$$
I\left(f_{1}, f_{2} ; \Delta t\right)=C_{12}\left\{\left|\eta_{1}\right|^{2} e^{-\Gamma_{L} \Delta t}+\left|\eta_{2}\right|^{2} e^{-\Gamma_{S} \Delta t}-2\left|\eta_{1}\right|\left|\eta_{2}\right| e^{-\frac{\left(\Gamma_{S}+\Gamma_{L}\right)}{2} \Delta t} \cos \left[\Delta m \Delta t+\phi_{2}-\phi_{1}\right]\right\}
$$

with $\Delta m=m_{L}-m_{S}$, and

$$
\eta_{i} \equiv\left|\eta_{i}\right| e^{i \phi_{i}}=\frac{\left\langle f_{i}|T| \mathrm{K}_{\mathrm{L}}\right\rangle}{\left\langle f_{i}|T| \mathrm{K}_{\mathrm{S}}\right\rangle} ; C_{12}=\frac{|\mathscr{N}|^{2}}{2\left(\Gamma_{S}+\Gamma_{L}\right)}\left|\left\langle f_{1}|T| \mathrm{K}_{\mathrm{S}}\right\rangle\left\langle f_{2}|T| \mathrm{K}_{\mathrm{S}}\right\rangle\right|^{2}
$$


Expression (2.2) is valid for $\Delta t \geq 0$, while for $\Delta t<0$ the substitutions $\Delta t \rightarrow|\Delta t|$ and $1 \leftrightarrow 2$ have to be applied. Due to the huge difference in the lifetimes of the physical states $\left(\tau_{L} \gg \tau_{S}\right)$, for $t_{1} \gg t_{2}, \tau_{S}$ (or $t_{2} \gg t_{1}, \tau_{S}$ ) the decay intensity in eq.(2.2) behaves like the initial state were an incoherent mixture of states $\left|\mathrm{K}_{\mathrm{S}}\right\rangle\left|\mathrm{K}_{\mathrm{L}}\right\rangle$ and $\left|\mathrm{K}_{\mathrm{L}}\right\rangle\left|\mathrm{K}_{\mathrm{S}}\right\rangle$. Hence the detection of a kaon at large times tags a $\mathrm{K}_{\mathrm{S}}$ in the opposite direction. This is a unique feature at a $\phi$-factory, not possible at fixed target experiments, that can be exploited to select pure $\mathrm{K}_{\mathrm{S}}$ beams.

\section{Measurement of $K_{S}$ semileptonic charge asymmetry}

At KLOE a $\mathrm{K}_{\mathrm{S}}$ is tagged by identifying the interaction of the $\mathrm{K}_{\mathrm{L}}$ in the calorimeter $\left(\mathrm{K}_{\mathrm{L}}{ }^{-}\right.$ crash). In fact about $50 \%$ of the produced $\mathrm{K}_{\mathrm{L}}$ 's in $\phi \rightarrow \mathrm{K}_{\mathrm{S}} \mathrm{K}_{\mathrm{L}}$ events reach the calorimeter before decaying; their associated interactions are identified by a high energy, neutral and delayed deposit in the calorimeter, i.e. not associated to any charged track in the event, and delayed of $\sim 30 \mathrm{~ns}$ (as $\beta_{K} \sim 0.22$ ) with respect to a photon coming from the interaction region. Pure $\mathrm{K}_{\mathrm{S}}$ samples have been selected exploiting this tagging technique. In particular $\mathrm{K}_{\mathrm{S}} \rightarrow \pi e v$ decays are selected requiring a $\mathrm{K}_{\mathrm{L}}$-crash and two tracks forming a vertex close to the IP, and associated with two energy deposits in the calorimeter. Pions and electrons are recognized using their TOF. A control sample of $\phi \rightarrow \mathrm{K}_{\mathrm{S}} \mathrm{K}_{\mathrm{L}} \rightarrow \pi^{0} \pi^{0}, \pi e v$ with the semileptonic decay close to the IP has been used to correct efficiencies evaluated with Monte Carlo. The analysis of a KLOE data sample corresponding to $1.7 \mathrm{fb}^{-1}$ yields a preliminary measurement of the $\mathrm{K}_{\mathrm{S}}$ semileptonic charge asymmetry:

$$
A_{S}=\left(-3.9 \pm 5.7_{-2.4}^{+3.3}\right) \times 10^{-3},
$$

improving by about a factor two the statistical uncertainty with respect to the previous KLOE result [8]. The measured $A_{S}$ value (3.1) is consistent with the expectation $A_{S}=A_{L} \simeq 3.3 \times 10^{-3}$ imposed by CPT invariance $\left(A_{\mathrm{L}}\right.$ is the $\mathrm{K}_{\mathrm{L}}$ semileptonic charge asymmetry and is precisely measured [9]), while its uncertainty is approaching the level necessary to reveal CP violation in the $\mathrm{K}_{\mathrm{S}}$ (i.e. $A_{S} \neq 0$ ). From the sum and the difference of $A_{S}$ and $A_{L}$ one can perform a CPT test. In fact using the values of $A_{L}, \Re \delta$, and $\Re \varepsilon^{1}$ from other experiments [9], the real part of the CPT violating and $\Delta S=\Delta Q$ violating (conserving) parameter $x_{-}(y)$ in semileptonic decay amplitudes can be evaluated:

$$
\begin{gathered}
\Re x_{-}=\frac{A_{S}-A_{L}}{4}-\Re \delta=(-2.1 \pm 1.6) \times 10^{-3} \\
\Re y=\Re \varepsilon-\frac{A_{S}+A_{L}}{4}=(1.8 \pm 1.6) \times 10^{-3},
\end{gathered}
$$

improving the uncertainty with respect to previous results [8]. The uncertainty on $A_{S}$ can be further reduced at the level of $\approx 3 \times 10^{-3}$ with the analysis of the full KLOE-2 data sample.

\section{Test of Time reversal and CPT symmetries in neutral kaon transition}

As explained in Refs [10, 11], exploiting the entanglement of the neutral kaon pair in the initial state (2.1), it is possible to exchange in and out states in a transition process. For instance it

\footnotetext{
${ }^{1} \varepsilon$ and $\delta$ are the usual complex parameters describing $C P$ and $C P T$ violation in the mixing of neutral kaons.
} 
is possible to observe the transition $\mathrm{K}^{0} \rightarrow \mathrm{K}_{-}$and its T-conjugated $\mathrm{K}_{-} \rightarrow \mathrm{K}^{0}$ or CPT-conjugated $\mathrm{K}_{-} \rightarrow \overline{\mathrm{K}}^{0}$ process, with $\mathrm{K}_{-}$defined as the state filtered by the decay into $3 \pi^{0}$, a pure $\mathrm{CP}=-1$ final state $^{2}$. Any deviation from unity of the corresponding ratios of probabilities

$$
\begin{aligned}
R_{2, \mathrm{~T}}(\Delta t) & =P\left[\mathrm{~K}^{0}(0) \rightarrow \mathrm{K}_{-}(\Delta t)\right] / P\left[\mathrm{~K}_{-}(0) \rightarrow \mathrm{K}^{0}(\Delta t)\right] \\
R_{2, \mathrm{CPT}}(\Delta t) & =P\left[\mathrm{~K}^{0}(0) \rightarrow \mathrm{K}_{-}(\Delta t)\right] / P\left[\mathrm{~K}_{-}(0) \rightarrow \overline{\mathrm{K}}^{0}(\Delta t)\right]
\end{aligned}
$$

would be an unambiguous signal of T or CPT violation, respectively. In this case the relevant observable quantities at a $\phi$-factory are the ratios of double decay intensities ${ }^{3}$ :

$$
R_{2, \mathrm{~T}}^{\exp }(\Delta t) \equiv \frac{I\left(\ell^{-}, 3 \pi^{0} ; \Delta t\right)}{I\left(\pi \pi, \ell^{+} ; \Delta t\right)} ; R_{2, \mathrm{CPT}}^{\exp }(\Delta t) \equiv \frac{I\left(\ell^{-}, 3 \pi^{0} ; \Delta t\right)}{I\left(\pi \pi, \ell^{-} ; \Delta t\right)} .
$$

In particular the observable double ratio:

$$
\frac{R_{2, C P T}^{\exp }\left(\Delta t \gg \tau_{S}\right)}{R_{4, C P T}^{\exp }\left(\Delta t \gg \tau_{S}\right)}=1-8 \Re \delta-8 \Re x_{-} .
$$

with $R_{4, \mathrm{CPT}}^{\exp }(\Delta t) \equiv \frac{I\left(\ell^{+}, 3 \pi^{0} ; \Delta t\right)}{I\left(\pi \pi, \ell^{+} ; \Delta t\right)}$, is predicted to be strictly equal to unity by CPT invariance, and constitutes one of the most robust and cleanest observable for a model independent CPT test that has never been performed in the neutral kaon system.

The analysis of KLOE and KLOE-2 data is ongoing and at an advanced stage, allowing to perform for the first time the test based on the double ratio (4.2) with a precision of $\mathscr{O}\left(10^{-3}\right)$.

\section{Search for the CP-violating $\mathrm{K}_{\mathrm{S}} \rightarrow 3 \pi^{0}$ decay}

The Standard Model prediction for the branching ratio of the CP-violating decay $\mathrm{K}_{\mathrm{S}} \rightarrow 3 \pi^{0}$ is $\mathrm{BR}\left(\mathrm{K}_{\mathrm{S}} \rightarrow 3 \pi^{0}\right) \sim 1.9 \times 10^{-9}$, making the direct observation of this decay quite a challenge. The best upper limit comes from the analysis of $1.7 \mathrm{fb}^{-1}$ collected by KLOE, searching for six photons coming from the IP and a $\mathrm{K}_{\mathrm{L}}$-crash [12]: $\mathrm{BR}\left(\mathrm{K}_{\mathrm{S}} \rightarrow 3 \pi^{0}\right)<2.6 \times 10^{-8}$ at $90 \%$ C.L. . This result can be further improved with the analysis of the additional $5 \mathrm{fb}^{-1}$ of data which are being collected by KLOE-2. A preliminary analysis of $300 \mathrm{pb}^{-1}$ shows the good quality of KLOE-2 data even in presence of a larger machine background with respect to KLOE. After hardening the selection criteria to get about ten times better background rejection, and after applying the scheme of the previous analysis [12], only one candidate event survives, leaving room to improvements and the possibility to reach a final sensitivity on the BR below $10^{-8}$.

\section{Conclusions}

The entangled neutral kaon system at DAФNE is an excellent laboratory for the study of discrete symmetries. While the analysis of the full KLOE data set is being completed, the KLOE-2 experiment at the upgraded DAFNE is currently taking data with the plan to collect $L \geq 5 \mathrm{fb}^{-1}$ by end of March 2018. The study of discrete symmetries with neutral kaons is one of the key issues at KLOE-2, and the precision of several tests performed by KLOE will be further improved.

\footnotetext{
${ }^{2} \mathrm{~K}_{-}$is assumed to be orthogonal to $\mathrm{K}_{+}$, the state filtered by the decay into $\pi \pi$, a pure $\mathrm{CP}=+1$ final state.

${ }^{3}$ In the following the semileptonic decays $\pi^{+} \ell^{-} v$ and $\pi^{-} \ell^{+} \bar{v}$ will be denoted for brevity as $\ell^{-}$and $\ell^{+}$, respectively.
} 


\section{References}

[1] A. Gallo et al.. DAFNE status report, Conf.Proc. C060626 (2006) 604-606, SLAC-PUB-12093.

[2] M. Adinolfi et al., KLOE collaboration, Nucl. Instr. and Meth. A 488 (2002) 51.

[3] M. Adinolfi et al., KLOE collaboration, Nucl. Instr. and Meth. A 482 (2002) 364.

[4] M. Zobov et al., Phys. Rev. Lett. 104, 174801 (2010).

[5] C. Milardi et al., JINST 7, 2012,T03002.

[6] G. Amelino-Camelia et al., Eur. Phys. J. C 68, 619 (2010).

[7] A. Di Domenico et al. , Handbook on Neutral Kaon Interferometry at a $\phi$-factory, Frascati Phys. Ser. 43 (2007).

[8] F. Ambrosino et al., KLOE collaboration, Phys. Lett. B 636 (2006) 173.

[9] C. Patrignani et al. (Particle Data Group), Chin. Phys. C 40, 100001 (2016).

[10] J. Bernabeu, A. Di Domenico, P. Villanueva-Perez, Nucl. Phys. B. 868, 102 (2013)

[11] J. Bernabeu, A. Di Domenico, P. Villanueva-Perez, JHEP 10, 139 (2015)

[12] D. Babusci et al., KLOE-2 collaboration, Phys. Lett. B 723, 54 (2013). 